\title{
Concise Communication
}

\section{Uptake in newly approved antibiotics prescribed to patients with carbapenem-resistant Enterobacterales (CRE)}

\author{
Katie J. Suda PharmD, MS, FCCP ${ }^{1,2}$, Alfredo Traversa PharmD ${ }^{3}$, Ursula Patel PharmD, BCPS, BCIDP, AAHIVP ${ }^{4}$, \\ Linda Poggensee $\mathrm{MS}^{5}$, Margaret A. Fitzpatrick MD, MS ${ }^{5,6}$, Geneva M. Wilson PhD, MPH ${ }^{5}$ and \\ Charlesnika T. Evans PhD, MPH ${ }^{5,7}$ \\ ${ }^{1}$ Department of Veterans' Affairs, Center for Health Equity Research and Promotion, Veterans' Health Administration (VA) Pittsburgh Healthcare System, \\ Pittsburgh, Pennsylvania, ${ }^{2}$ Division of General Internal Medicine, Department of Medicine, University of Pittsburgh School of Medicine, Pittsburgh, Pennsylvania, \\ ${ }^{3}$ Internal Medicine, Rush University Medical Center, Chicago, Illinois, ${ }^{4}$ Department of Veterans' Affairs, Edward Hines Jr VA Hospital, Hines, Illinois, ${ }^{5}$ Department \\ of Veterans' Affairs, Center of Innovation for Complex Chronic Healthcare, Edward Hines Jr VA Hospital, Hines, Illinois, ${ }^{6}$ Division of Infectious Diseases, \\ Department of Medicine, Loyola University Chicago Stritch School of Medicine, Maywood, Illinois and ${ }^{7}$ Center for Health Services and Outcomes Research, \\ Department of Preventive Medicine Institute for Public Health and Medicine, Northwestern University Feinberg School of Medicine, Chicago, Illinois
}

\begin{abstract}
We assessed trends in treatment of patients with CRE from 2012 through 2018. We detected decreased utilization of aminoglycosides and colistin and increased utilization in extended-spectrum cephalosporins and ceftazidime-avibactam. We found significant uptake of ceftazidime-avibactam, a newly approved antibiotic, to treat CRE infections.
\end{abstract}

(Received 12 June 2021; accepted 16 September 2021; electronically published 24 November 2021)

Carbapenem-resistant Enterobacterales (CRE) is classified as an "urgent threat" pathogen. ${ }^{1}$ In the United States, the reported CRE incidence is $0.3-2.93$ infections per 100,000 person years, with the highest incidence occurring in long-term acute-care facilities. ${ }^{2}$ Historically, polymyxins and tigecycline had been considered the drugs of choice for CRE infections. The use of these agents has been complicated by toxicity, limited efficacy, and suboptimal pharmacokinetics. $^{3}$ A recent survey of US hospital-based pharmacists showed that new anti-CRE antibiotics, including ceftazidimeavibactam, are positioned as first-line agents for CRE bacteremia $>80 \%$ of the time. ${ }^{4}$ Nevertheless, one study identified lower inpatient utilization of ceftazidime-avibactam. However, this study extrapolated antibiotic purchase data to national CRE estimates and was unable to describe patient-specific treatment. ${ }^{4}$ The objective of this study was to describe trends in treatment for patients with cultures positive for CRE.

\section{Materials and methods}

This cross-sectional study of CRE treatments was conducted across 134 Veterans' Health Administration (VA) facilities from 2012 through 2018. Patients were identified through the VA Corporate Data Warehouse (CDW). Patients were included in the study if they were aged $\geq 18$ years, had a positive CRE culture, and received a gram-negative antibiotic.

Author for correspondence: Dr Katie J. Suda, E-mail: ksuda@pitt.edu

Cite this article: Suda KJ, et al. (2023). Uptake in newly approved antibiotics prescribed to patients with carbapenem-resistant Enterobacterales (CRE). Infection Control \& Hospital Epidemiology, 44: 674-677, https://doi.org/10.1017/ice.2021.452
CRE cultures were defined as E. coli, Klebsiella spp, or Enterobacter spp isolates that were either positive for carbapenemase production by a phenotypic method or were nonsusceptible to imipenem, meropenem, or doripenem. Cultures were categorized either as bloodstream infections (BSIs) or non-BSI cultures, including urine, respiratory, and other cultures. Patients receiving antimicrobials were defined as receiving $\geq 24$ hours of antibiotics 2 days before through 5 days after the CRE culture. The VA has a national drug formulary. After FDA approval in February 2015, ceftazidime-avibactam was added to the formulary, restricted to infectious disease and facility-authorized personnel. Personnel are notified of new additions to the formulary through listservs, education, key program offices (eg, infectious disease), and field representatives.

Continuous data were evaluated with independent $t$ tests and 2-way Wilcoxon tests for parametric and nonparametric data, respectively. Categorical data was assessed with a $\chi^{2}$ test. Poisson regression was applied as a trend test to assess changes in CRE treatment over time stratified by culture source. Two-sided $P$ values $<.05$ were considered significant. SAS version 9.4 software (SAS Institute, Cary, NC) was used for all analyses.

\section{Results}

In total, 7,767 patients with positive CRE cultures were identified; $65 \%(\mathrm{~N}=5,082$ cultures representing 2,772 unique patients at 111 of 134 facilities) met the definition of receiving antibiotic treatment and were included in the cohort (Table 1). Urine cultures were the major culture source $(51.7 \%)$ of CRE, followed by respiratory

(c) The Author(s), 2021. Published by Cambridge University Press on behalf of The Society for Healthcare Epidemiology of America. This is an Open Access article, distributed under the terms of the Creative Commons Attribution licence (http://creativecommons.org/licenses/by/4.0/), which permits unrestricted re-use, distribution and reproduction, provided the original article is properly cited. 
Table 1. Patient Demographic and Medical Characteristics of carbapenem-resistant Enterobacterales (CRE) Cultures Overall and Stratified by Bloodstream Infection (BSI) and Non-BSI

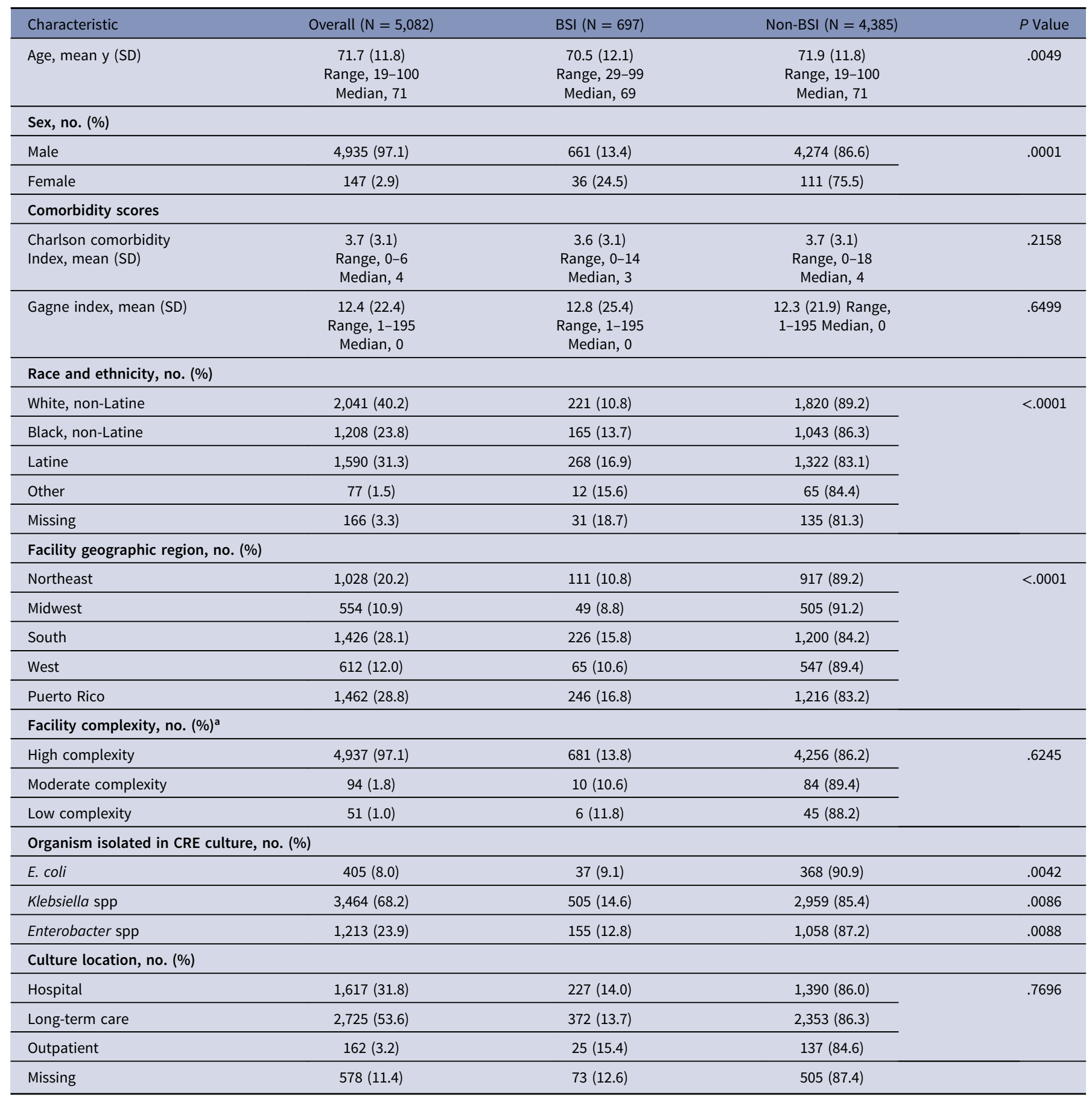

${ }^{a}$ VA facilities are classified by complexity: levels 1a-c were high complexity, level 2 was moderate complexity, and level 3 was low complexity. Facility complexity is based on patient characteristics, clinical programs, and teaching programs.

(20.1\%), blood (13.7\%), and other (14.5\%). Most (97.1\%) cultures were identified in high complexity facilities, most commonly in the southern United States (28.1\%) and Puerto Rico (28.8\%). The BSI group was significantly younger than the non-BSI group, but comorbidity scores were similar (Table 1). African Americans and Latines were significantly more likely to have CRE BSI cultures than whites. The frequency of BSI CRE isolates increased significantly (Supplementary Table 1 online).
In total, 4,385 patients with non-BSI CRE cultures received antibiotics: $37.7 \%$ received fluoroquinolones, $35.0 \%$ received extended-spectrum cephalosporins (cefepime and ceftazidime), $29.8 \%$ received penicillins, $29.4 \%$ received carbapenems, $21.2 \%$ received aminoglycosides, $18.8 \%$ received polymyxins, and $4.2 \%$ received ceftazidime-avibactam. (Percentages exceed 100\% because $36 \%$ received multiple antibiotics.) Over the study period, we detected decreased utilization of aminoglycosides $(-49.0 \%$; 


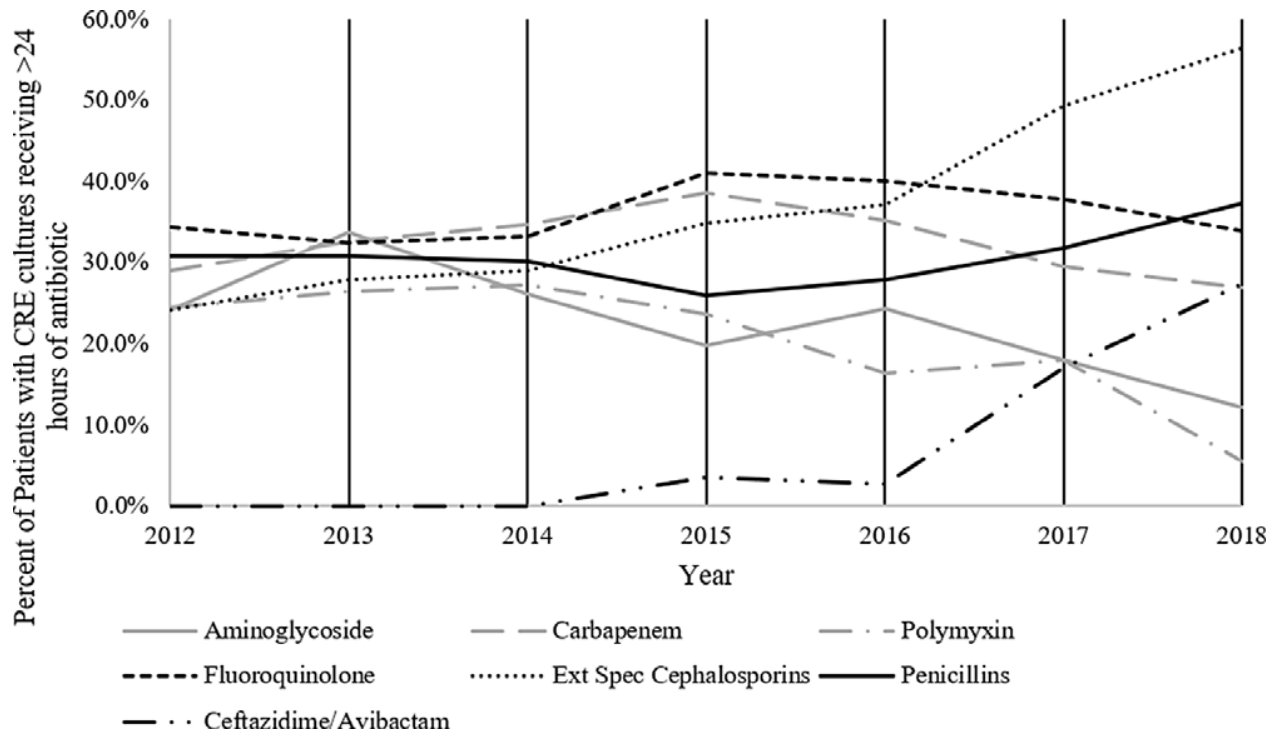

Fig. 1. Trends in antibiotics prescribed to veterans with non-BSI cultures positive for carbapenem-resistant Enterobacterales (CRE). $P$ values for trend: aminoglycosides <.0001; carbapenems 0.7985 ; polymyxins $<.0001$; fluoroquinolones $<.0001$; extended spectrum cephalosporins $<.0001$; penicillins 0.4559 ; and ceftazidimeavibactam. Ceftazidime-avibactam was approved by the US Food and Drug Administration February 2015 and was available for distribution April 2015. Ceftazidime-avibactam was added to the national VA formulary in December 2015 restricted to infectious disease specialists or facility-authorized provider (when infectious disease specialists are not available at the individual facility). Extended-spectrum cephalosporins included third- and fourthgeneration cephalosporins; only ceftazidime and cefepime were prescribed. Cefiderocol was not FDA approved during the study period.

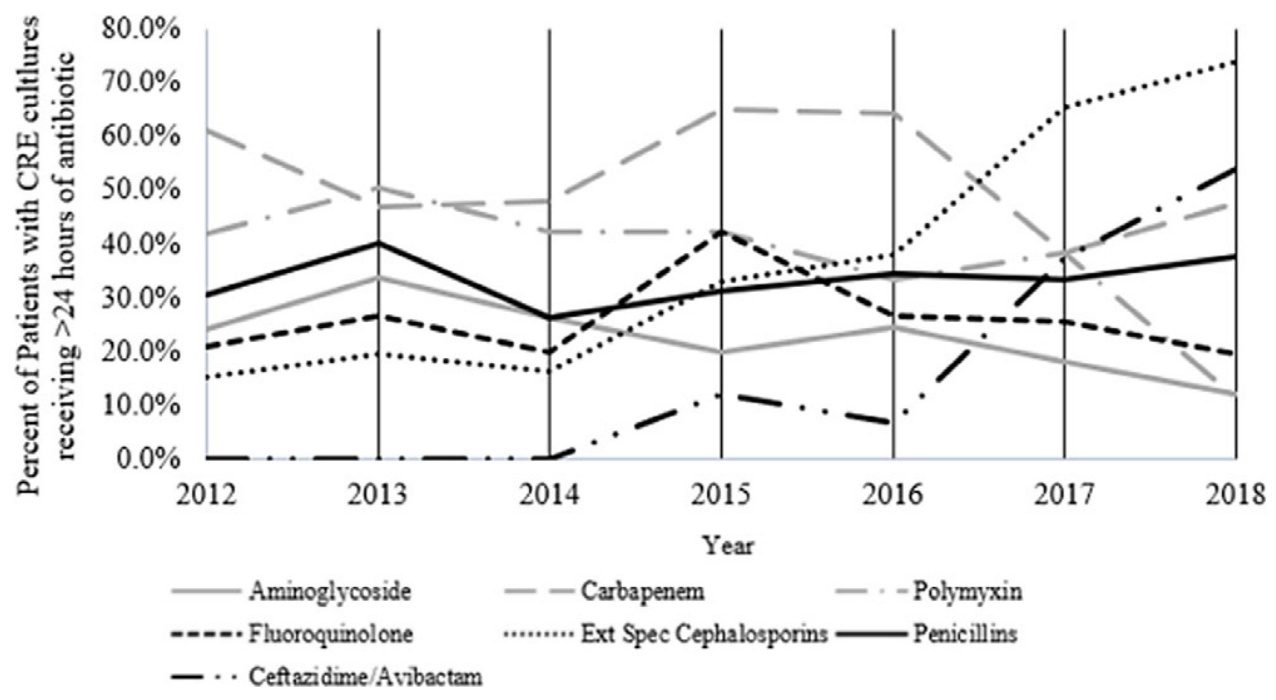

Fig. 2. Trends in antibiotics prescribed to veterans with BSI cultures positive for carbapenemresistant Enterobacterales (CRE). $P$ value for trends: aminoglycosides 0.0026 ; carbapenems 0.4741; polymyxins .0019; fluoroquinolones .6411 ; extended spectrum cephalosporins <.0001; penicillins .7110; and ceftazidime-avibactam. ${ }^{2}$ Ceftazidime-avibactam was approved by the US Food and Drug Administration February 2015 and was available for distribution April 2015. Ceftazidime-avibactam was added to the national VA formulary in December 2015 restricted to infectious disease specialists or facility-authorized provider (when infectious disease specialists are not available at the individual facility). ${ }^{3} \quad$ Extended-spectrum cephalosporins included third- and fourth-generation cephalosporins; only ceftazidime and cefepime were prescribed. Cefiderocol was not FDA approved during the study period.
$P<.001$ for trend) and polymyxins $(-79.7 \% ; P<.001)$ and increases in extended-spectrum cephalosporins $(111.1 \% ; P<.001)$ and ceftazidime-avibactam $(433.0 \% ; P<.001)$ (Fig. 1). In 2018 (the last study year), $22.7 \%$ of CRE non-BSI patients received ceftazidime-avibactam and $4.5 \%$ received polymyxins (vs $22.2 \%$ in 2012 ).

Among 697 with CRE BSI, 53.4\% received carbapenems, $40.3 \%$ received aminoglycosides, $39.3 \%$ received polymyxins, $32.9 \%$ received penicillins, $32.6 \%$ received extended-spectrum cephalosporins, $26.1 \%$ received fluoroquinolones, and $11.6 \%$ received ceftazidime-avibactam. Over the study period, we detected decreases in aminoglycosides $(-58.0 \% ; P<.0026$ for trend) and polymyxins $(-72.6 \% ; P<.002)$ and increases in extended-spectrum cephalosporins $(385.5 \% ; P<.001)$ and ceftazidime-avibactam $(154 \%$; $P<.001$ ) (Fig. 2). In 2018, $54.1 \%$ of CRE BSI patients received ceftazidime-avibactam and $11.9 \%$ received polymyxins (vs $41.9 \%$ in 2012).

\section{Discussion}

This national study was the first to evaluate prescribing trends in patients with confirmed CRE. In the non-BSI and BSI groups, ceftazidime-avibactam use increased dramatically and aminoglycoside and polymyxin use decreased. The significant increase in ceftazidime-avibactam is consistent with the results of a recently published national survey of Infectious Diseases pharmacists, where ceftazidime-avibactam was preferred to polymyxins for CRE. ${ }^{4}$ Likewise, national hospital claims demonstrated increases in ceftazidime-avibactam and decreases in polymyxins; however, in contrast to this study, these authors did not confirm that patients had positive CRE cultures. ${ }^{5}$ Finally, these results are consistent with IDSA guidance on the treatment of resistant gram-negative infections, which recommend ceftazidime-avibactam and other newer $\beta$-lactam antibiotics for CRE infections. ${ }^{6}$

Interestingly, extended-spectrum cephalosporins also increased, likely empiric use prior to confirmed CRE, and may reflect a shift from empiric use of anti-pseudomonal penicillins. Such shifts may have been prompted by increasing recognition during our study period of nephrotoxicity risk with the empiric antibiotic combination of vancomycin and piperacillin-tazobactam.

In this study, persons with CRE-BSI were younger than those with other infection sources. These results echo other findings 
showing that those with BSI were younger than those with hospital- or ventilator-associated pneumonia or complicated urinary tract infections. Additionally, the rate of secondary comorbidities was similar between those with BSI versus other infection types. ${ }^{7}$

Given toxicity concerns, decreases in aminoglycosides and polymyxins were expected. Retrospective studies have demonstrated the association of acute kidney injury (AKI) with polymyxins and aminoglycosides. Observational studies have found improved safety and effectiveness with ceftazidime-avibactam compared with polymyxins. ${ }^{8}$ A meta-analysis that evaluated the use of ceftazidime-avibactam in patients with MDR gram-negative infections found the pooled clinical success rate to be $73.3 \%$, similar to polymyxins $(66 \%-79 \%))^{9}$

This study had several limitations. Therapies included in this study could have been used for different organisms, which may have overestimated true utilization rates of these antibiotics for CRE. For non-BSI, we were unable to determine definitively whether positive CRE cultures reflected colonization or true infection. Furthermore, because we did not capture antibiotics prescribed $>5$ days after culture, we may have underestimated ceftazidime-avibactam use, an agent not typically prescribed until a multidrug-resistant organism (MDRO) is identified. This study was conducted in the Veterans Health Administration (VA), which may not be generalizable. Also, other factors may have limited antibiotic utilization, such as drug shortages.

This large national cohort study of veterans with CRE showed an encouraging trend toward increased uptake and utilization of ceftazidime-avibactam for CRE, with decreased utilization of "older" agents such as aminoglycosides and polymyxins. These results are consistent with IDSA and VA guidance, which now recommend ceftazidime-avibactam among the first-line treatments for severe CRE infections. Future studies will need to assess uptake and utilization of other, more recently approved antibiotics targeting multidrug-resistant gram-negative bacteria.

Acknowledgments. The opinions expressed are those of the authors and do not represent those of the Department of Veterans' Affairs or the US government.

Financial support. This work was supported by The Department of Veterans' Affairs, Veterans' Health Administration, Office of Research and Development,
Health Services Research and Development (grant no. IIR 16-028); by the Rehabilitation Research and Development Career Development Award (grant no. B2826-W to M.A.F.); and by a Research Career Scientist Award (grant no. RCS 20-192 to C.T.E).

Conflicts of interest. All authors report no conflicts of interests or financial disclosures relevant to this article.

Supplementary material. To view supplementary material for this article, please visit https://doi.org/10.1017/ice.2021.452

\section{References}

1. Centers for Disease Control and Prevention. Antibiotic resistance threats in the United States, 2013. Centers for Disease Control and Prevention website. https://www.cdc.gov/drugresistance/pdf/ar-threats-2013-508.pdf. Accessed September 5, 2019.

2. Livorsi D, Chorazy M, Schweizer M, et al. A systematic review of the epidemiology of carbapenem-resistant Enterobacteriaceae in the United States. Antimicrob Resist Infect Control 2018;7:55.

3. Thaden J, Pogue J, Kaye K. Role of newer and re-emerging older agents in the treatment of infections caused by carbapenem-resistant. Enterobacteriaceae. 2017;8:403-416.

4. Clancy CJ, Potoski BA, Buehrle D, Nguyen MH. Estimating the treatment of carbapenem-resistant Enterobacteriaceae infections in the United States using antibiotic prescription data. Open Forum Infect Dis 2019;6(8):ofz344.

5. Strich JR, Ricotta E, Warner S, et al. Pharmacoepidemiology of ceftazidimeavibactam use: a retrospective cohort analysis of 210 US hospitals. Clin Infect Dis 2021;72:611-621.

6. Infectious Diseases Society of America. Infectious Diseases Society of America guidance on the treatment of antimicrobial resistant gram-negative infections. https://www.idsociety.org/practice-guideline/amr-guidance/. Published 2020. Accessed November 4. 2021.

7. Pang F, Jia XQ, Zhao QG, Zhang Y. Factors associated to prevalence and treatment of carbapenem-resistant Enterobacteriaceae infections: a sevenyear retrospective study in three tertiary-care hospitals. Ann Clin Microbiol Antimicrob 2018;17:13.

8. van Duin D, Lok JJ, Earley M, et al. Colistin versus ceftazidime-avibactam in the treatment of infections due to carbapenem-resistant Enterobacteriaceae. Clin Infect Dis 2018;66:163-171.

9. Wilson GM, Fitzpatrick M, Walding K, et al. Meta-analysis of clinical outcomes using ceftazidime/avibactam, ceftolozane/tazobactam, and meropenem/vaborbactam for the treatment of multidrug-resistant gram-negative infections. Open Forum Infect Dis 2021;8(2):ofaa651. 